\title{
Genetic and biological characterization of a densovirus isolate that affects dengue virus infection
}

\author{
Ana Luiza Pamplona Mosimann', Juliano Bordignon', Giovanny Camacho Antevêre Mazzarotto', \\ Maria Cristina M Motta ${ }^{2}$, Federico Hoffmann ${ }^{3}$, Claudia Nunes Duarte dos Santos ${ }^{1 /+}$ \\ ${ }^{1}$ Instituto Carlos Chagas-Fiocruz, Rua Prof. Algacyr Munhoz Mader 3775, Curitiba, PR, Brasil \\ ${ }^{2}$ Instituto de Biofísica Carlos Chagas Filho, Universidade Federal do Rio de Janeiro, Rio de Janeiro, RJ, Brasil \\ ${ }^{3}$ Department of Biochenisty and Molecular Biology, Mississippi State University, MS, USA
}

Brevidensoviruses have an encapsidated, single-stranded DNA genome that predominantly has a negative polarity. In recent years, they have received particular attention due to their potential role in the biological control of pathogenic arboviruses and to their unnoticed presence in cell cultures as contaminants. In addition, brevidensoviruses may also be useful as viral vectors. This study describes the first genetic and biological characterization of a mosquito densovirus that was isolated in Brazil; moreover, we examined the phylogenetic relationship between this isolate and the other brevidensoviruses. We further demonstrate that this densovirus has the potential to be used to biologically control dengue virus (DENV) infection with in vitro co-infection experiments. The present study provides evidence that this densovirus isolate is a fast-spreading virus that affects cell growth and DENV infection.

Key words: densovirus - Brevidensovirus - biological control - dengue virus

Over the past several decades, a new set of viruses that are non-pathogenic to humans have been isolated from mosquito vectors, such as Aedes sp. (Kittayapong et al. 1999, Jousset et al. 2000). Arbovirology researchers are particularly interested in these viruses because of their potential use as biological controls and expression vectors (Afanasiev et al. 1994, Carlson et al. 2006, Wei et al. 2006). In this study, we identified and molecularly characterised a mosquito densovirus (MDV) that belongs to the Brevidensovirus genus of the Densovirinae sub-family, which belongs to the Parvoviridae family (Bergoin \& Tijssen 2000). MDVs are non-enveloped viruses that have a single-stranded DNA genome of approximately $4 \mathrm{~kb}$ and predominantly negative polarity $(85 \%)$, packaged within the capsid (Afanasiev et al. 1994). Viruses belonging to this genus have three major open reading frames in the positive polarity strand, which encode for non-structural (NS1 and NS2) and structural proteins (VP1 and VP2). These genomic sequences possess self-complementary palindromic sequences at their 5' and 3' ends, which are essential for the replication and encapsidation of their genetic material (Afanasiev et al. 1994). These sequences differ between the various brevidensoviruses in terms of their length and sequence pairing generates a $\mathrm{Y}$ or $\mathrm{T}$ shaped structure (Bergoin $\&$ Tijssen 2000). These viruses are thought to be non-pathogenic to humans, but they show varying degrees of pathogenicity in mosquitoes (O'Neill et al. 1995, Kittayapong et al. 1999, Carlson et al. 2006, Suchman et al. 2006).

Financial support: FIOCRUZ, CNPq, CNPq/PROSUL, Fundação Araucária ALPM is supported by a CNPq fellowship. CNDS is a CNPq fellowship recipient.

+Corresponding author: clsantos@tecpar.br

Received 29 September 2010

Accepted 19 January 2011
In this paper, we isolated and characterised a MDV that was a contaminant in $\mathrm{C} 6 / 36$ cell cultures infected with yellow fever virus. Our results from in vitro coinfection assays with this MDV and a recent clinical isolate of dengue virus (DENV) indicate that the MDV interfered with dengue viral morphogenesis.

\section{MATERIALS AND METHODS}

Cells and viruses - The Aedes albopictus cell line (Igarashi 1978) (C6/36, ATCC, CRL-1660) was cultured in Leibovitz's medium (L-15, Gibco/Invitrogen, Grand Island, NY, USA) supplemented with 5\% fetal bovine serum (FBS) (Gibco/Invitrogen, Grand Island, NY, USA), 0.26\% tryptose (Sigma-Aldrich, St. Louis, MO, USA) and $25 \mu \mathrm{g} /$ $\mathrm{mL}$ gentamicin (Gibco/Invitrogen, Grand Island, NY, USA) at $28^{\circ} \mathrm{C}$. Kidney epithelial cells from Cercopithecus aethiops (Vero E6, ATCC, CRL-1586) were maintained at $37^{\circ} \mathrm{C}$ with $5 \% \mathrm{CO}_{2}$ in Iscove's Modified Dulbecco's medium (Gibco/Invitrogen, Grand Island, NY, EUA) supplemented with $5 \% \mathrm{FBS}$ and $25 \mu \mathrm{g} / \mathrm{mL}$ gentamicin.

The MDV stocks, which are referred to as BR/07 MDV, were prepared by infecting C6/36 cell cultures.

BR DEN3 290-02, which is a recent clinical isolate of DENV serotype 3 (DENV-3), was used for the coinfection experiments (GenBank accession EF629369) (Nogueira et al. 2008). The viral stock that was used for the experiments was the supernatant from the fifth passage of the $\mathrm{C} 6 / 36$ cell cultures.

The YF17DD virus was kindly provided by Dr Ricardo Galler [Biomanguinhos, Oswaldo Cruz Foundation (Fiocruz), Rio de Janeiro]; the viral supernatants used in the experiments were prepared through serial passage in Vero cells.

Total nucleic acid extraction - The total cellular nucleic acid content was extracted from the cells by phenol extraction as follows. The cells were lysed by three cycles of freeze/thaw. The cell debris was removed by centrifu- 
gation (10 min at $400 \mathrm{~g}$ ) and sodium dodecyl sulfate and $\beta$-mercaptoethanol were added to the supernatant at a final concentration of $1 \%$. Following incubation for $3 \mathrm{~min}$ at $72^{\circ} \mathrm{C}$, an equal volume of phenol:chlorophorm:isoamylalcohol (25:24:1) was added. The aqueous phase was removed and re-extracted with an equal volume of chlorophorm:isoamyl-alcohol (24:1). The nucleic acids were precipitated with $10 \% 3 \mathrm{M}$ sodium acetate, $\mathrm{pH}$ 5.3, and 2.5 volumes of ethanol; the extracted nucleic acids were finally resuspended in nuclease-free water.

Panchip - The nucleic acids that were extracted from the $C 6 / 36$ cell line were randomly amplified and hybridized to a pan-viral DNA microarray, as described by Wang et al. (2003).

Transmission electron microscopy - For routine transmission electron microscopy, the cells were fixed for $1 \mathrm{~h}$ in $2.5 \%$ glutaraldehyde and diluted in $0.1 \mathrm{M}$ cacodylate buffer, $\mathrm{pH}$ 7.2. The samples were washed twice with the same buffer and subsequently fixed in $1 \% \mathrm{OsO}_{4}, 0.8 \%$ $\mathrm{KFe}(\mathrm{CN})_{6}$ and $5 \mathrm{mM} \mathrm{CaCl}_{2}$ diluted in $0.1 \mathrm{M}$ cacodylate buffer. After fixation, the cells were washed, dehydrated in a graded series of acetone solutions and embedded in Epoxy resin. Ultrathin sections were stained with uranyl acetate and lead citrate before analysis with a Zeiss 900 transmission electron microscope.

MDV isolation - BR/07 MDV was initially identified in a cell culture supernatant sample that was also infected with yellow fever virus (BR/01). A neutralization assay with yellow fever virus polyclonal antibodies was performed to isolate the MDV. The sample was serially diluted $\left(10^{-1}-10^{-7}\right)$ in L-15 medium supplemented with $0.26 \%$ tryptose and $25 \mu \mathrm{g} / \mathrm{mL}$ gentamicin to a final volume of $500 \mu \mathrm{L}$. A yellow fever polyclonal antibody
(50 $\mu \mathrm{L}$ ) was then added to each dilution and the mixture was incubated for $2 \mathrm{~h}$ at $37^{\circ} \mathrm{C}$. Following the incubation period, each mixture was used to infect $3.5 \times 10^{5} \mathrm{C} 6 / 36$ cells. After incubation for $1 \mathrm{~h}$ at $28^{\circ} \mathrm{C}$, the inoculum was discarded and replaced with fresh medium. Five days after infection, the total cellular nucleic acid content was extracted from the cells.

Yellow fever reverse transcription-polymerase chain reaction (RT-PCR) and MDV PCR - Yellow fever virus $(\mathrm{BR} / 01)$ infection of the samples was assessed with reverse transcription of the total nucleic acid extract. An ImProm-II ${ }^{\mathrm{TM}}$ Reverse Transcription System (Promega, Madison, WI, USA) with random hexamers (Invitrogen, Carlsbad, CA, USA) was used according to manufacturer's instructions. The synthesised cDNA and the total nucleic acid were tested with specific yellow fever and MDV primers, respectively, in a PCR reaction with recombinant Taq DNA polymerase (Invitrogen, Carlsbad, CA, USA) according to the manufacturer's instructions. The primers used for the PCR amplification were YF21, YF34, DNV3F and DNV3R (Table I). Positive and negative samples were included for each reaction.

MDV genome amplification and sequencing Whole genome PCR amplification was used to genetically characterise BR/07 MDV. We used the following high-fidelity enzymes according to the manufacturer's instructions: Platinum ${ }^{\circledR}$ Taq DNA Polymerase High Fidelity (Invitrogen, Carlsbad, CA, USA), QIAGEN ${ }^{\circledR}$ LongRange PCR System (QIAGEN, Valencia, CA, USA) and TripleMaster ${ }^{\circledR}$ PCR System (Eppendorf, Westbury, NY, USA). The primers were initially designed based on the Ae. albopictus densovirus sequence (GenBank accession NC_004285) and subsequently from the BR/07

TABLE I

Primers used in reverse transcription-polymerase chain reaction (PCR), PCR and sequencing

\begin{tabular}{lcc}
\hline Primer & Sequence 5' $\rightarrow 3^{\prime}$ & Position \\
\hline DNV1F & TGTTGGGAGCATGACGCACAGT & $375-396^{a}$ \\
DNV1R & GGTTCTCTGTCTGCGTCTGCGATGAACA & $3072-3045^{a}$ \\
DNV2F & TCGTGATACGGATACTCCAAGATACAG & $43-69^{a}$ \\
DNV3F & AATCGAGAAACAGCATACTACACATTCGT & $1134-1162^{a}$ \\
DNV3R & TTTATTTCCATAGATATTGACTGTTTCGAT & $1457-1428^{a}$ \\
DNV4F & TATAAGTCATATTCCATATAAGAAATATTATT & $10-42^{a}$ \\
DNV4R & ACGGTTGATATACACGTTCCATCA & $913-890^{a}$ \\
DNV5F & GGAGAAGACAACAGCAAAACAGCAA & $1860-1884^{a}$ \\
DNV5R & CTGGTGTTATCATTGGTTCTTCAAATAGGA \\
DNV6F & TACTTGGACTTCAACTACGTATCAAATCA & $2163-2134^{a}$ \\
DNV6R & TTCTGAGGTGGAATACGGAGGTGGA & $2783-2811^{a}$ \\
DNV7F & CACAACCACAAATTCCAGACGAAACC & $3791-3767^{a}$ \\
DNV7R & CAACGCAGACTGAATTCATCACTCGAT & $3438-3463^{a}$ \\
DNV8R & GGCTCAATGAGAGTTGGAGGACGACT & $327-301^{a}$ \\
YF21 & CACATATCCTAGGTATTGTAAGCC & $2437-2412^{a}$ \\
YF34 & GCCAATGAGGCTGTCCAAGACC & $9193-9170^{b}$ \\
\end{tabular}

$a$ : GenBank accession: GU452720; $b$ : GenBank accession: DQ100292. 
MDV nucleotide sequence as it became available. The primer sequences are listed in Table I. To amplify the 5' and 3' ends of BR/07 MDV, the total nucleic acid extract from the supernatant of infected cell cultures was denatured at $95^{\circ} \mathrm{C}$ for $5 \mathrm{~min}$, ligated with T4 RNA ligase (New England Biolabs, Ipswich, MA, USA) at $37^{\circ} \mathrm{C}$ for $30 \mathrm{~min}$ and incubated at $16^{\circ} \mathrm{C}$ for $16 \mathrm{~h}$. The ligated product was purified by phenol extraction as previously described. The purified DNA was used as the template for a PCR reaction with the DNV7F and DNV7R primers. The PCR product was purified with a High Pure PCR Product Purification Kit (Roche Diagnostics, Mannheim, Alemanha) and was inserted into a pGEM ${ }^{\mathbb{R}}$-T-Easy vector (Promega, Madison, WI, USA) for nucleotide sequencing.

The nucleotide sequencing was performed with a BigDye $^{\circledR}$ Terminator v3.1 Cycle Sequencing Kit (Applied Biosystems, Foster City, IA, USA) in an ABI PRISM $^{\circledR} 3100$ Genetic Analyzer (Applied Biosystems, Foster City, IA, USA). Alternatively, the PCR products were sequenced by Macrogen Inc (Seoul, Korea). The sequences were assembled with the phred/Phrap/consed software package (www.phrap.org) (Ewing et al. 1998, Ewing \& Green 1998, Gordon et al. 1998, 2001). The final consensus sequence was deposited in GenBank under the accession GU452720.

Secondary structure prediction for the 5' and 3' ends - The DNA mfold server (Zuker 2003) (www.bioinfo.rpi. edu/applications/mfold/cgi-bin/dna-form1.cgi) was used to predict the secondary structure of the BR/07 MDV 5 ' and 3' ends. For comparison, the same procedure was used to predict the secondary structure of Aedes densonucleosis virus (GenBank accession M37899), which has previously been described (Afanasiev et al. 1991). The default settings were used for all of the analyses.

Phylogenetic analysis - Bioinformatic searches for similar sequences were performed online with the National Center for Biotechnology Information Blast server. Based upon the results, we assembled a reference panel of densovirus sequences from GenBank (Table II). Sequences ranging from the start codon of the open reading frame (ORF) on the $5^{\prime}$ end to the stop codon of the ORF on the 3' end were aligned using the ClustalW algorithm (Thompson et al. 1994); distance calculations and phylogenetic analysis were performed using Molecular Evolutionary Genetics Analysis version 4 (Tamura et al. 2007). The pairwise distance comparisons were based on the proportion of nucleotide differences between the sequences and phylogenies were estimated by neighbour-joining (Saitou \& Nei 1987) using the maximum composite likelihood distance (Tamura \& Kumar 2002). Support for the nodes was evaluated with 1,000 bootstrap pseudoreplicates (Felsenstein 1985).

Polyclonal and monoclonal antibody production Adult $\mathrm{BALB} / \mathrm{c}$ mice were immunised five times with viable BR/07 MDV virus particles obtained from the supernatant of $\mathrm{C} 6 / 36$ infected cell cultures. Prior to immunisation, a pre-immune serum sample was collected. On day zero, the mice were immunised intraperitoneally with a $1: 1$ mixture $(200 \mu \mathrm{L})$ of virus and Freund's complete adjuvant (Sigma-Aldrich, St. Louis,
MO, USA). On days seven, 14 and 21, the mice were immunised intraperitoneally with a 1:1 mixture (200 $\mu \mathrm{L}$ ) of virus and Alu-Gel-S (SERVA Electrophoresis, Heidelberg, Germany). Finally, on day 28, the mice were immunised intravenously and intraperitoneally with virus only (100 $\mu \mathrm{L} /$ route). Three days after the last immunisation, a post-immune serum sample and the spleen were collected. The serum was evaluated with an indirect immunofluorescence assay (IFA) with infected cell cultures to validate its use as a source of a polyclonal antibody (Supplementary data). The spleen was processed for monoclonal antibody production as described by Mazzarotto et al. (2009). Our protocol was approved by the Ethical Committee on Animal Experiments of Fiocruz (LW-26/09).

$M D V$ concentration - Viral stock titration was done by quantitative PCR as described by Ledermann et al. (2004). DNA from the viral supernatants was purified with an AxyPrep Body Fluid Viral DNA/RNA Miniprep Kit (Axygen Biosciences, Union City, CA, USA) and quantified in an ABI 7500 thermocycler/fluorescence reader with a TaqMan Universal PCR Master Mix (Applied Biosystems, Foster City, IA, USA). Standard curves were constructed using serial 10 -fold dilutions $\left(10^{10}-10^{1}\right.$ genome copies) of the plasmid T\&A cloning vector (Real

\section{TABLE II}

Densovirus sequences used in phylogenetic analysis

GenBank

accession

Identification

EF579760 Culex pipiens pallens densovirus strain XJ057

EF579763 Cux. pipiens pallens densovirus strain XJ0511

EF579762 Cux. pipiens pallens densovirus strain XJ059

EF579764 Cux. pipiens pallens densovirus strain XJ0545

EF579770 Cux. pipiens pallens densovirus strain YN05169

EF579758 Cux. pipiens pallens densovirus strain GZWN2

EF579759 Cux. pipiens pallens densovirus strain GZWN5

EF579761 Cux. pipiens pallens densovirus strain XJ058

EF579769 Cux. pipiens pallens densovirus strain YN05159

EF579765 Cux. pipiens pallens densovirus strain YN0569

EF579768 Cux. pipiens pallens densovirus strain YN05152

EF579767 Cux. pipiens pallens densovirus strain YN05150

EF579757 Cux. pipiens pallens densovirus strain GZWN1

EF579766 Cux. pipiens pallens densovirus strain YN05145

EF579771 Cux. pipiens pallens densovirus strain YN05217

EF579756 Cux. pipiens pallens densovirus strain JZ-16

EU233812 Anopheles gambiae densonucleosis virus

NC_011317 An. gambiae densonucleosis virus

M37899

AY095351

AY310877

AY605055

NC 004285

X74945
Aedes densonucleosis virus

Aedes albopictus 66/36 cell densovirus

Ae. albopictus $\mathrm{C} 6 / 36$ cell densovirus

Haemagogus equinus densovirus

Ae. albopictus densovirus

Ae. albopictus parvovirus 
Biotech Corporation, Banqiao, Taiwan) with the PCR insert, which was cloned according to the manufacturer's instructions. The plasmid molecular weight was calculated with the sequence manipulation software available at http://www.bioinformatics.org/sms2/dna_mw.html. The plasmid was purified with a Wizard Plus SV Miniprep kit (Promega, Madison, WI, USA), the concentration was estimated with a Nano Drop ND-1,000 Spectrophotometer (Nano Drop technologies, Wilmington, DE, USA) and the number of copies was calculated according to Ke et al. (2006). The number of copies was calculated by taking into account that our target consists of single-stranded DNA, although double-stranded DNA was used for the standard curve.

Cell growth and cell-cycle kinetics - Six-well plates were seeded with $4 \times 10^{4} \mathrm{C} 6 / 36 \mathrm{cells} / \mathrm{cm}^{2}$ and incubated for $24 \mathrm{~h}$; the cells were subsequently infected with a multiplicity of four genomes/cell [4 multiplicity of genome (MOG)] of BR/07 MDV or mock infected. At 24, 48 and $72 \mathrm{~h}$ post-infection, the cells were detached, stained with trypan blue and the number of viable cells was counted with a Neubauer chamber. The same conditions were used to evaluate the cell cycle stage via flow cytometry, as described by Fried et al. (1978).

Co-infection experiments - C6/36 cell cultures were infected with a multiplicity of infection (MOI) of one or 10 of DENV-3 (BR DEN3 290-02), four or 0.04 MOG of BR/07 MDV or a mixture of these two viruses. Non-infected cultures were used as the negative control (mock). The extent of infection was evaluated at 24, 48 and $72 \mathrm{~h}$ with flow cytometry in a BD FACSCalibur ${ }^{\mathrm{TM}}$ Flow Cytometer (BD Biosciences, San Jose, CA, USA), as well as by titration of the DENV particles from the cell culture supernatants. The titration was performed using the focus immunodetection technique (Desprès et al. 1993); flow cytometry was performed as previously described by Bordignon et al. (2002). The results were analysed with the FlowJo flow cytometry analysis software (TreeStar, Inc, Ashland, OR, USA).

Replicon experiments - C6/36 cell cultures prepared and infected with BR/07 MDV (3 MOG or 0.03 MOG), as described above, were subsequently transfected with the BR DEN3 290-02 replicon (Mosimann et al. 2010). Five days post-infection/transfection, the monolayer was fixed and stained for immunofluorescence analysis with either anti-MDV or anti-DENV-3 polyclonal antibodies and the percentage of positive cells was estimated.

Virus spread experiments - The C6/36 cells were seeded at a density of $5 \times 10^{4}$ cells $/ \mathrm{cm}^{2}$ and incubated for $48 \mathrm{~h}$; the cells were subsequently infected with 0.6 or $0.006 \mathrm{MOG}$ of BR/07 MDV (or a mock infection for the control). After incubation for $1 \mathrm{~h}$ at $28^{\circ} \mathrm{C}$, the inoculum was removed and cells were washed with phosphate buffered saline (PBS) buffer before replenishment with cell medium. At various time points $(0,1,3,5,7,9,11 \mathrm{~h}$ and $24 \mathrm{~h}$ ), the cells were either detached and immunolabeled for flow cytometry analysis or fixed and permeabilized with a $1: 1$ solution of methanol:acetone at $-20^{\circ} \mathrm{C}$ for at least $2 \mathrm{~h}$ for an indirect immunofluorescence assay.
IFA - The C6/36 cell monolayers that were previously fixed and permeabilised were incubated with mouse anti-MDV polyclonal antibodies at $37^{\circ} \mathrm{C}$ for $45 \mathrm{~min}$, washed three times with PBS and incubated for $45 \mathrm{~min}$ at $37^{\circ} \mathrm{C}$ with goat anti-mouse conjugated to fluorescein isothiocyanate diluted 1:100 in PBS containing $0.3 \%$ (v/v) of $10 \mu \mathrm{g} / \mathrm{mL}$ Evan's blue. The cells were subsequently incubated at room temperature for $5 \mathrm{~min}$ with $1 \mathrm{mg} / \mathrm{mL}$ 4'-6-diamidine-2-phenyl indole, washed five times with PBS buffer and, once dry, were overlaid with a solution of PBS and $10 \%(\mathrm{v} / \mathrm{v})$ glycerol.

The immunofluorescence images were captured with a Nikon Eclipse TE300 microscope (Nikon, Tokyo, Japan) attached to a CoolSNAP ${ }^{\text {TM }}-$ Pro $_{\text {cf }}$ camera (Media Cybernetics, Bethesda, MD, EUA). The images were visualised and edited using the Image-Pro ${ }^{\circledR}$ PLUS v.4.5.1.29 software (Media Cybernetics, Bethesda, MD, EUA).

Statistics - The results are expressed as the mean \pm standard deviation of the data. Differences between the groups were analysed with a one-way or two-way analysis of variance, followed by a Bonferroni post-test. A p value $<0.05$ was considered significant.

\section{RESULTS}

The BR/07 MDV was initially detected in the supernatant of C6/36 cells infected with YFV (BR/01) that had been sent to our laboratory for research purposes (Supplementary data). Because of the atypical cytopathic effect that was observed in the $\mathrm{C} 6 / 36$ cell cultures, we suspected that there was additional microorganism contamination. A putative contaminating virus was identified by electron microscopyt, which showed viral particles of various sizes within the same cell. Hybridization of randomly amplified nucleic acids from the culture to a pan-viral DNA microarray yielded strong hybridisation for multiple oligonucleotide probes from known densoviruses. Viral identification was later confirmed by PCR using primers for conserved regions of the Brevidensovirus genome, which were retrieved from the public data banks (Supplementary data).

The BR/07 MDV was subsequently isolated by eliminating the yellow fever virus, which was also present in the sample, by neutralization. BR/07 MDV isolation was confirmed by RT-PCR and PCR with yellow fever and MDVspecific primers, respectively (Supplementary data).

Genomic sequencing indicated that the BR/07 MDV isolate contains three major ORFs, which is consistent with previous descriptions of other viruses within this genus. The left ORF is 2,550 nucleotides (nt), the mid ORF is 1,089 nt and the right ORF 1,077 nt (Fig. 1A). Nucleotide sequence alignments indicate that BR/07 MDV is closely related to a densovirus that was previously isolated from Aedes aegypti (GenBank accession M37899). When the two predicted coding sequences were compared for these two viruses, we found 101 nucleotide substitutions and 38 amino acid replacements for BR/07 MDV. The relationship between these viruses was confirmed by a neighbour-joining phylogeny, which grouped them together with $100 \%$ bootstrap support (Fig. 1B). 
Due to the limited amount of sequence data available for the 5' and 3' ends of these viruses, it was difficult to predict the first and last nt of the BR/07 MDV genome. However, the BR/07 MDV end sequences that were defined by the genome assembly display the predicted "Y"-shaped structure, similar to the predicted structure for Ae. densonucleosis virus, although with longer arms (Supplementary data). The arms formed by the 5' and 3' end of the BR/07 MDV isolate were 108 and $164 \mathrm{nt}$ long, respectively, whereas the analogous structures for $A e$. densonucleosis virus are 102 and $146 \mathrm{nt}$ long.

Our initial biological characterization of the BR/07 MDV isolate involved assessment of the cell growth and cell cycle kinetics. Preliminary observations indicated that infection with this virus affected cell growth. Infected $\mathrm{C} 6 / 36$ cell growth was significantly inhibited relative to the non-infected cell growth (Fig. 2A). Cell cycle kinetic experiments showed that the infected cells were arrested in the $\mathrm{G} 2$ phase (Fig. 2B).

Our next step was to quantify the viral inoculum. We tried several titration protocols for the BR/07 MDV isolate, including the $\mathrm{TCID}_{50}$ protocol described by Jousset et al. (1993), or the focus immunodetection technique

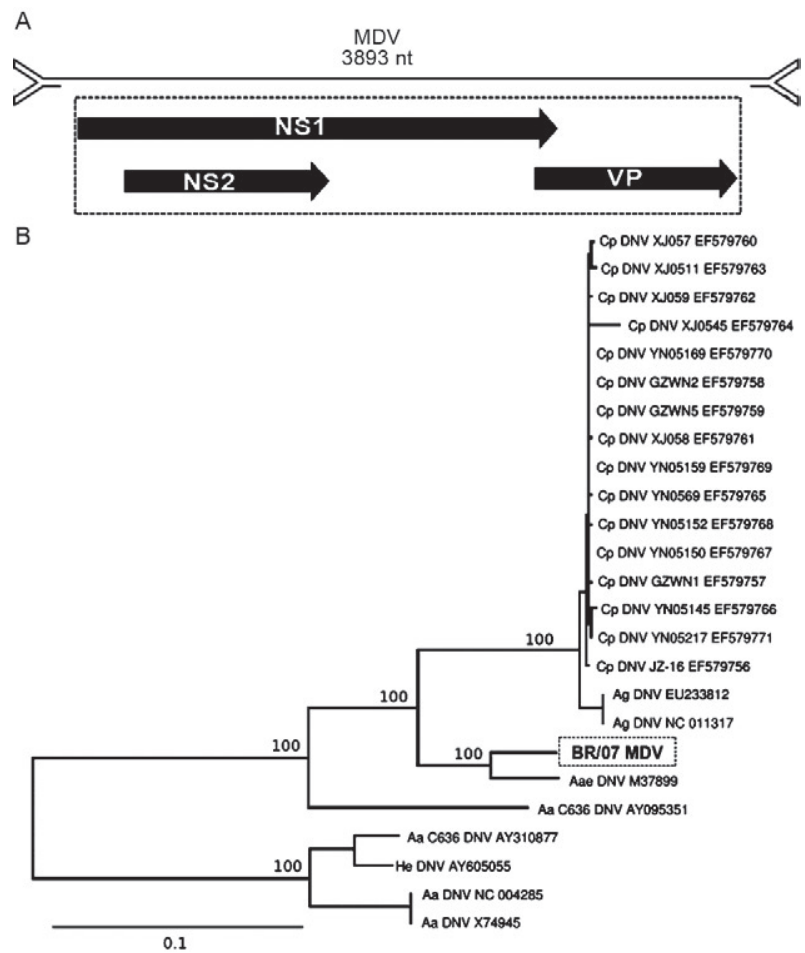

Fig. 1A: schematic representation of the BR/07 mosquito densovirus (MDV) viral isolate genome (above) and its open reading frames (below) (drawn with Vector NTI software from Vector NTI Advance 10.3.1 Software package, Invitrogen, Carlsbad, CA, USA); B: phylogenetic tree of viruses belonging to the Brevidensovirus genus. The tree was generated based on the region contained within the dashed box in A. Aa: Aedes albopictus; Aae: Aedes aegypti; Ag: Anopheles gambiae; Cp: Culex pipiens; He: Haemagogus equinus; NS: nonstructural; VP: structural proteins.
(Desprès et al. 1993), which were both unsuccessful. The number of genome copies was quantified through quantitative PCR as described by Ledermann et al. (2004).

The observations made while attempting to titrate the virus suggest that the BR/07 MDV infection spread in the culture within a few hours. To evaluate the rate of viral spread, an experiment was performed with two different virus concentrations ( $0.6 \mathrm{MOG}$ and $0.006 \mathrm{MOG})$. The results of these experiments (Fig. 3A) clearly show that the BR/07 MDV infection spread rapidly. At an MOG of 0.6, we were able to detect protein synthesis in the infected cells as early as $3 \mathrm{~h}$ post-infection (white arrows) and by $7 \mathrm{~h}$ postinfection, the whole monolayer was infected. This process was delayed with $0.006 \mathrm{MOG}$, and the whole monolayer was infected by $9 \mathrm{~h}$ post-infection. These IFA results are consistent with the flow cytometry data (Fig. 3B).

Several previous studies (Jousset et al. 1993, Carlson et al. 2006, Suchman et al. 2006, Wei et al. 2006) have shown that MDV can be used as a biological control for arboviral infection. Based on these observations, we evaluated the capacity of BR/07 MDV to affect the in vitro infection time course with a clinical isolate of dengue virus. Co-infection experiments were performed with either 4 MOG or $0.04 \mathrm{MOG}$ of BR/07 MDV in combination with DENV-3 at a MOI of one or 10. We found that there was significant inhibition of DENV infection, which was measured by the percentage of infected cells, irrespective of the MOI of DENV used when the cells were co-infected at $0.04 \mathrm{MOG}$ of BR/07 MDV (Fig. 4A, B). In contrast, there was no difference in the percentage of DENV infection in cells co-infected at $4 \mathrm{MOG}$ of BR/07 MDV and cells infected exclusively with DENV-3. Titration of DENV in the supernatants of the cultures infected with 4 MOG of

A

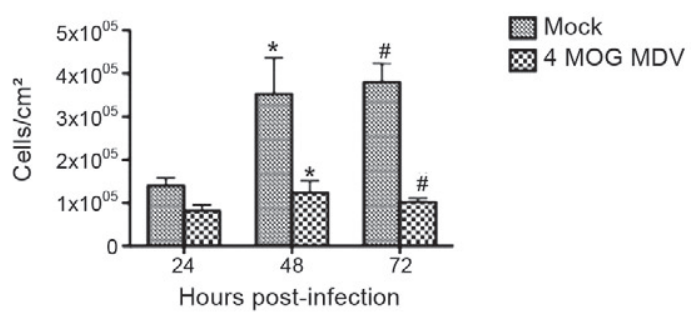

B

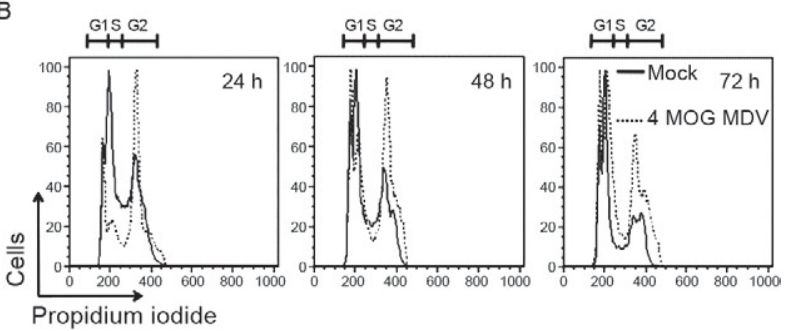

Fig. 2: cell growth and cell-cycle kinetics. A: cell growth. Results are expressed as the average number of cells $/ \mathrm{cm}^{2} \pm$ standard deviation of two experiments with two biological replicates each $(*: p$ $<0.01 ; \#: \mathrm{p}<0.001)$; B: cell-cycle kinetics. Results are from one representative of two experiments performed. MOG: multiplicity of genome; MDV: mosquito densovirus. 
BR/07 MDV (Fig. 4C) typically had lower values at $24 \mathrm{~h}$ and $48 \mathrm{~h}$ post-infection, although these observations were not statistically significant. At $72 \mathrm{~h}$ post-infection, a significant decrease in the DENV-3 viral titre was observed when the cells were co-infected with BR/07 MDV at both MOIs tested (4 MOG and 0.04 MOG).

To determine whether BR/07 MDV infection interfered with DENV-3 replication, we transfected MDVinfected C6/36 cells with a DENV-3 replicon (BR DEN3 290-02 replicon) and estimated the number of antiDENV-3 reactive cells at five days post-infection/transfection. The results suggest that (Supplementary data) there was no significant difference in the percentage of anti-DENV-3 reactive cells for the MDV infected and non-infected cells that were subsequently transfected with the DENV-3 replicon.

\section{DISCUSSION}

The main purpose of this work was to genetically and biologically characterise a new Brevidensovirus isolate (BR/07 MDV), as well as to assess its ability to affect DENV morphogenesis. BR/07 MDV was first detected in a C6/36 cell culture infected with an YFV sample $(\mathrm{BR} / 01)$ that was sent to our laboratory for research purposes. The atypical YFV phenotype in the mosquito cell culture suggested the presence of a contaminating co-infecting microorganism. Electron microscopy, microarray hybridisation, PCR and genome sequencing identified this microorganism as a MDV.

Based on the previously published data (Gorziglia et al. 1980, Jousset et al. 1993, O'Neill et al. 1995, Chen et al. 2004), we hypothesize that the cell line initially used in the YFV isolation, most likely a mosquito cell line, may have been contaminated. Unfortunately, because the cell culture supernatant sample was sent seven years ago for research purposes, there is no way to track the infection origin.

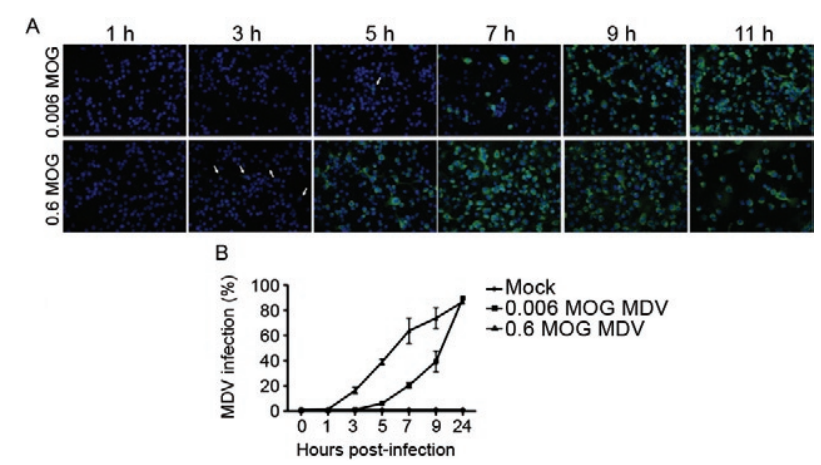

Fig. 3: virus spread experiments. A: indirect immunofluorescence assay of $\mathrm{C} 6 / 36$ cultures infected at 0.006 or 0.6 multiplicity of genomes (MOG) of BR/07 mosquito densovirus (MDV). Cells were fixed and incubated with anti-MDV polyclonal antibody followed by anti-mouse conjugated to fluorescein isothiocyanate. Cell nuclei were stained with 4'-6-diamidine-2-phenyl indole. Images were obtained with a 400X magnification factor; B: flow cytometry results of C6/36 cultures infected at 0.006 or $0.6 \mathrm{MOG}$ of BR/07 MDV. For flow cytometry, cells were immunolabeled with anti-MDV polyclonal antibody followed by anti-mouse conjugated to phycoeritrin. Results are expressed as means \pm standard deviation of three experiments.
Thus, we suggest that researchers who work with arbovirus isolation from mosquitoes should check their cell lines periodically for MDV infection. The potential of these viruses to pass unnoticed in cell cultures is due to their ability to establish persistent infections that are not associated with any obvious cytopathic effect (Gorziglia et al. 1980, Jousset et al. 1993, O'Neill et al. 1995).

Phylogenetic analysis grouped BR/07 MDV with $A e$. aegypti densovirus, which was supported by high bootstrap values (Fig. 1). This could be due to the fact that the viral isolate reported in this article was clearly present as a cell culture contaminant, without means of tracing its real origin. Similar results were described by Chen et al. (2004).

Previous findings by Paterson et al. (2005) suggest that the expression of Ae. aegypti densonucleosis virus NS1 in C6/36 cells causes the cells to halt in the G2 phase. Consistent with this, we saw that BR/07 MDV affected Ae. albopictus cell growth (Fig. 2A) and appeared to cause cell cycle arrest in G2 (Fig. 2B). Cell cycle arrest has also been previously described by Op De Beeck and Caillet-Fauquet (1997) in a study with another parvovirus, the Minute virus of mice. This study showed that NS1 interferes with cellular DNA replication and induces chromatin damage. Similarly, Oleksiewicz and Alexandersen (1997) saw that infection of Crandell feline kidney cells with Aleutian mink disease parvovirus induced cell-cycle arrest.

Previous studies have also suggested that MDV reduces the severity of DENV infection (Burivong et al. 2004). Thus, we tested the ability of MDV to act as a biological control for DENV infection in vitro with co-infection experiments. We observed significantly lower levels of cell infection and a lower virus titre in C6/36 cells that were co-infected with BR/07 MDV and DENV-3 compared to cells infected with DENV-3 alone. However, the discrepancy observed between the DENV titre results (Fig. 4C) and the percentage of infected cells determined with flow cytometry (Fig. 4A, B) remains unclear. Although co-infection at $4 \mathrm{MOG}$ of BR/07 MDV did not significantly inhibit dengue infection (Fig. 4A, B), DENV-3 viral progeny generation was significantly inhibited by BR/07 MDV at both MOG tested $72 \mathrm{~h}$ post co-infection (Fig. 4C). Therefore, the effect of BR/07 MDV on DENV infection does not appear to be related to viral entry into the cells or genome replication/translation but may be due to changes in viral assembly or budding. Replicons are especially well suited to study viral replication once they have all the elements needed for the replication of the viral genetic material in cells, but they do not encode the functional structural proteins and consequently are not able to generate new viral particles (Jones et al. 2005). We therefore used a DENV-3 replicon (Mosimann et al. 2010) in co-infection experiments with BR/07 MDV as described above to confirm our findings. DENV-3 replication was not significantly affected by MDV infection (3 MOG or $0.03 \mathrm{MOG}$ ) (Supplementary data). However, because the percentage of transfected cells was low, the possibility that MDV infection interferes with DENV-3 replication cannot be completely ruled out. 
A

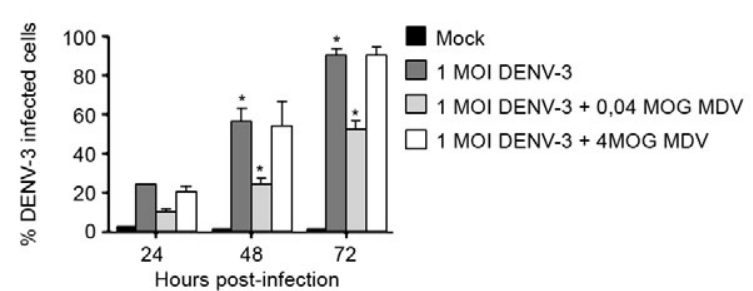

B

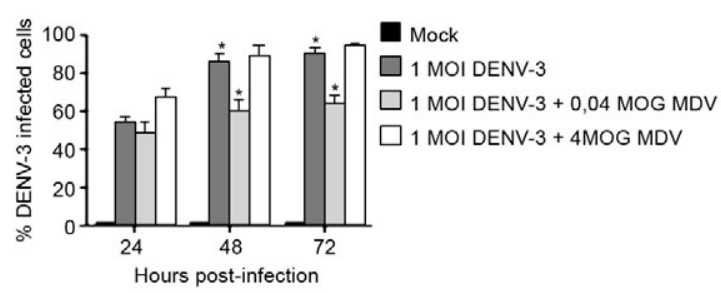

C

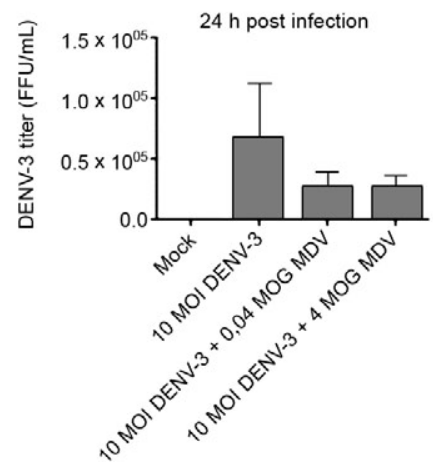

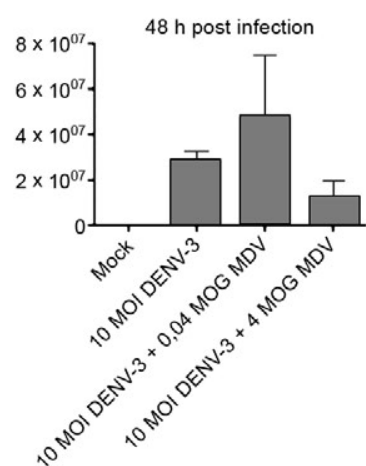

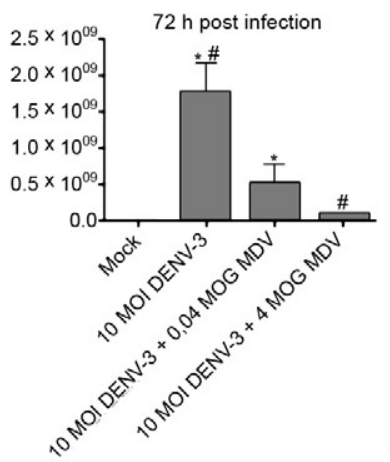

Fig. 4: co-infection experiments. A: flow cytometry on cells co-infected with one multiplicity of infection (MOI) of dengue virus-3 (DENV-3) and 0.04 multiplicity of genomes (MOG) or $4 \mathrm{MOG}$ of BR/07 mosquito densovirus (MDV); B: flow cytometry on cells co-infected with $10 \mathrm{MOI}$ DENV-3 and 0.04 MOG or 4 MOG of MDV; C: DENV-3 titers in the supernatants of co-infected cultures (\#/*: $p<0.001)$. Results are expressed as means \pm standard deviation of three experiments. FFU: focus forming units.

Wei et al. (2006) obtained promising results for the use of MDV as a biological control. Specifically, they observed that DENV-2 infection was 100 -fold decreased in MDV-co-infected Ae. albopictus mosquitoes, compared to mosquitoes infected with DENV-2 alone. Using a different approach, Burivong et al. (2004) showed that $\mathrm{C} 6 / 36$ cells that were persistently infected with Ae. albopictus densovirus had a less severe infection, although they were not protected from infection itself. Significantly decreased DENV-2 viral particle production was observed in cells that were persistently infected with MDV at $72 \mathrm{~h}$ and $96 \mathrm{~h}$ but was not observed at $120 \mathrm{~h}$ post-infection. However, the use of different co-infection protocols makes it difficult to compare the previous findings with our own.

Viruses that belong to the Densovirus genus display certain features that make them suitable for use as biological controls. They are non-enveloped viruses that can survive for long periods of time in nature. They also act as larvicides and can be vertically transmitted to mosquito offspring. Additionally, infection in adult mosquitoes has been associated with a decreased lifespan (Jousset et al. 1993, Carlson et al. 2006, Suchman et al. 2006). Because these viruses possess a small genome, they may also prove useful as expression vectors, although this remains to be confirmed (Afanasiev et al. 1994). They may also be useful for immunisation strategies, where sequences that are able to interfere with either the replication of clinically important arboviruses or the reproduction of mosquito vectors could be inserted into the MDV genome (Carlson et al. 2006). However, such possibilities must be addressed carefully, because previous findings have suggested that arthropod cells can have balanced, persistent infections with two or three heterologous viruses (Burivong et al. 2004, Kanthong et al. 2008, 2010).

\section{ACKNOWLEDGEMENTS}

To Paulo Arauco and Vanessa Stella, for their technical help with sequencing and cell culture procedures, respectively, to Dr Ricardo Galler, for providing the YF17DD strain, to Dr David Wang, for assistance with the microarray analysis, to Dr Eleonora Campos, for helpful discussion, and to Dr André Báfica, for critical reading.

\section{REFERENCES}

Afanasiev BN, Galyov EE, Buchatsky LP, Kozlov YV 1991. Nucleotide sequence and genomic organization of Aedes densonucleosis virus. Virology 185: 323-336.

Afanasiev BN, Kozlov YV, Carlson JO, Beaty BJ 1994. Densovirus of Aedes aegypti as an expression vector in mosquito cells. Exp Parasitol 79: 322-339.

Bergoin M, Tijssen P 2000. Molecular biology of Densovirinae. In S Faisst, J Rommelaere (eds.), Parvoviruses from molecular biology to pathology and therapeutic uses, Karger, Basel, p. 12-32.

Bordignon J, Pires Ferreira SC, Medeiros Caporale GM, Carrieri ML, Kotait I, Lima HC, Zanetti CR 2002. Flow cytometry assay for intracellular rabies virus detection. J Virol Methods 105: 181-186.

Burivong P, Pattanakitsakul SN, Thongrungkiat S, Malasit P, Flegel TW 2004. Markedly reduced severity of dengue virus infection in mosquito cell cultures persistently infected with Aedes albopictus densovirus (AalDNV). Virology 329: 261-269.

Carlson J, Suchman E, Buchatsky L 2006. Densoviruses for control and genetic manipulation of mosquitoes. Adv Virus Res 68: 361-392.

Chen S, Cheng L, Zhang Q, Lin W, Lu X, Brannan J, Zhou ZH, Zhang J 2004. Genetic, biochemical and structural characterization of a new densovirus isolated from chronically infected Aedes albopictus C6/36 cell line. Virology 318: 123-133.

Desprès P, Frenkiel MP, Deubel V 1993. Differences between cell membrane fusion activities of two dengue type-1 isolates reflect modifications of viral structure. Virology 196: 209-219. 
Ewing B, Green P 1998. Base-calling of automated sequencer traces using phred. II. Error probabilities. Genome Res 8: 186-194.

Ewing B, Hillier L, Wendl MC, Green P 1998. Base-calling of automated sequencer traces using phred. I. Accuracy assessment. Genome Res 8: 175-185.

Felsenstein J 1985. Confidence limits on phylogenies: an approach using the bootstrap. Evolution 39: 783-791.

Fried J, Perez AG, Clarkson BD 1978. Rapid hypotonic method for flow cytofluorometry of monolayer cell cultures. Some pitfalls in staining and data analysis. J Histochem Cytochem 26: 921-933.

Gordon D, Abajian C, Green P 1998. Consed: a graphical tool for sequence finishing. Genome Res 8: 195-202.

Gordon D, Desmarais C, Green P 2001. Automated finishing with autofinish. Genome Res 11: 614-625.

Gorziglia M, Botero L, Gil F, Esparza J 1980. Preliminary characterization of virus-like particles in a mosquito (Aedes pseudoscutellaris) cell line (Mos. 61). Intervirology 13: 232-240.

Igarashi A 1978. Isolation of a Singh's Aedes albopictus cell clone sensitive to dengue and Chikungunya viruses. J Gen Virol 40: 531-544.

Jones CT, Patkar CG, Kuhn RJ 2005. Construction and applications of yellow fever virus replicons. Virology 331: 247-259.

Jousset FX, Baquerizo E, Bergoin M 2000. A new densovirus isolated from the mosquito Culex pipiens (Diptera: Culicidae). Virus Res 67: 11-16.

Jousset FX, Barreau C, Boublik Y, Cornet M 1993. A parvo-like virus persistently infecting a C6/36 clone of Aedes albopictus mosquito cell line and pathogenic for Aedes aegypti larvae. Virus Res 29: 99-114.

Kanthong N, Khemnu N, Pattanakitsakul SN, Malasit P, Flegel TW 2010. Persistent, triple-virus co-infections in mosquito cells. $B M C$ Microbiol 10: 14.

Kanthong N, Khemnu N, Sriurairatana S, Pattanakitsakul S, Malasit P, Flegel TW 2008. Mosquito cells accommodate balanced, persistent co-infections with a densovirus and dengue virus. Dev Comp Immunol 32: 1063-1075.

Ke GM, Cheng HL, Ke LY, Ji WT, Chulu JL, Liao MH, Chang TJ, Liu HJ 2006. Development of a quantitative Light Cycler real-time RTPCR for detection of avian reovirus. J Virol Methods 133: 6-13.

Kittayapong P, Baisley KJ, O’Neill SL 1999. A mosquito densovirus infecting Aedes aegypti and Aedes albopictus from Thailand. Am J Trop Med Hyg 61: 612-617.

Ledermann JP, Suchman EL, Black WC 4th, Carlson JO 2004. Infection and pathogenicity of the mosquito densoviruses AeDNV, HeDNV and APeDNV in Aedes aegypti mosquitoes (Diptera: Culicidae). J Econ Entomol 97: 1828-1835.

Mazzarotto GA, Raboni SM, Stella V, Carstensen S, de Noronha L, Levis S, Zanluca C, Zanetti CR, Bordignon J, Duarte dos Santos CN 2009. Production and characterization of monoclonal anti- bodies against the recombinant nucleoprotein of Araucaria hantavirus. J Virol Methods 162: 96-100.

Mosimann AL, de Borba L, Bordignon J, Mason PW, dos Santos CN 2010. Construction and characterization of a stable subgenomic replicon system of a Brazilian dengue virus type 3 strain (BR DEN3 290-02). J Virol Methods 163: 147-152.

Nogueira MB, Stella V, Bordignon J, Batista WC, de Borba L, Silva LHP, Hoffmann FG, Probst CM, Santos CND 2008. Evidence for the co-circulation of dengue virus type 3 genotypes III and $\mathrm{V}$ in the Northern Region of Brazil during the 2002-2004 epidemics. Mem Inst Oswaldo Cruz 103: 483-488.

Oleksiewicz MB, Alexandersen S 1997. S-phase-dependent cell cycle disturbances caused by Aleutian mink disease parvovirus. $J$ Virol 71: 1386-1396

O'Neill SL, Kittayapong P, Braig HR, Andreadis TG, Gonzalez JP, Tesh RB 1995. Insect densoviruses may be widespread in mosquito cell lines. J Gen Virol 76: 2067-2074.

Op De Beeck A, Caillet-Fauquet P 1997. The NS1 protein of the autonomous parvovirus Minute virus of mice blocks cellular DNA replication: a consequence of lesions to the chromatin? J Virol 71: 5323-5329.

Paterson A, Robinson E, Suchman E, Afanasiev B, Carlson J 2005. Mosquito densonucleosis viruses cause dramatically different infection phenotypes in the C6/36 Aedes albopictus cell line. Virology 337: 253-261.

Saitou N, Nei M 1987. The neighbor-joining method: a new method for reconstructing phylogenetic trees. Mol Biol Evol 4: 406-425.

Suchman EL, Kononko A, Plake E, Doehling M, Kleker B, Black WC 4th, Buchatsky L, Carlson J 2006. Effects of AeDNV infection on Aedes aegypti lifespan and reproduction. Biol Control 39: 465-473.

Tamura K, Dudley J, Nei M, Kumar S 2007. MEGA4: Molecular Evolutionary Genetics Analysis (MEGA) software version 4.0. Mol Biol Evol 24: 1596-1599.

Tamura K, Kumar S 2002. Evolutionary distance estimation under heterogeneous substitution pattern among lineages. Mol Biol Evol 19: 1727-1736.

Thompson JD, Higgins DG, Gibson TJ 1994. CLUSTALW: improving the sensitivity of progressive multiple sequence alignment through sequence weighting, position-specific gap penalties and weight matrix choice. Nucleic Acids Res 22: 4673-4680.

Wang D, Urisman A, Liu YT, Springer M, Ksiazek TG, Erdman DD, Mardis ER, Hickenbotham M, Magrini V, Eldred J, Latreille JP, Wilson RK, Ganem D, DeRisi JL 2003. Viral discovery and sequence recovery using DNA microarrays. PLoS Biol 1: E2.

Wei W, Shao D, Huang X, Li J, Chen H, Zhang Q, Zhang J 2006. The pathogenicity of mosquito densovirus (C6/36DNV) and its interaction with dengue virus type II in Aedes albopictus. Am J Trop Med Hyg 75: 1118-1126.

Zuker M 2003. Mfold web server for nucleic acid folding and hybridization prediction. Nucleic Acids Res 31: 3406-3415. 


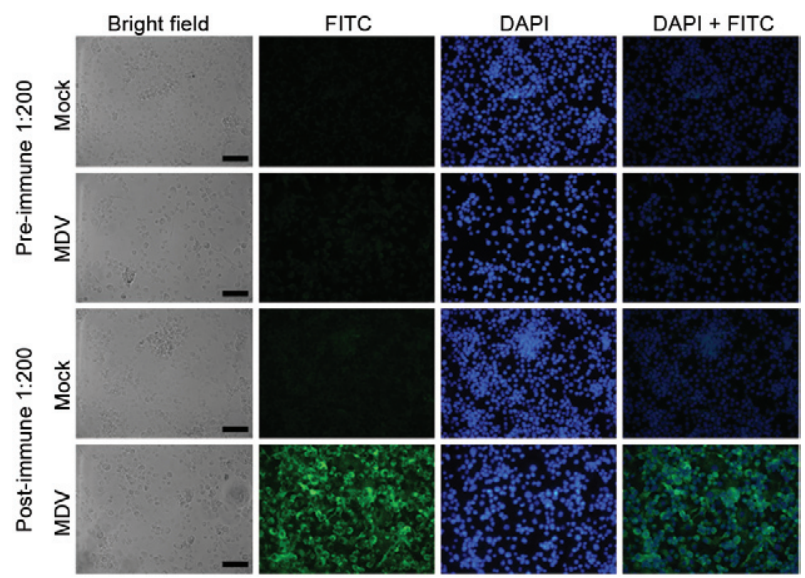

Characterization of anti-mosquito densovirus (MDV) polyclonal antibodies. Indirect immunofluorescence assay (IFA) of $\mathrm{C} 6 / 36$ cultures infected with MDV or mock infected. For IFA, the cells were fixed and incubated with pre or post-immune serum diluted 1:200 followed by anti-mouse conjugated to fluorescein isothiocyanate. Cell nuclei were stained with 4'-6-diamidine-2-phenyl indole (DAPI). Pictures were taken with $400 \mathrm{X}$ magnification factor. The scale bar in the bright field images corresponds to $50 \mu \mathrm{m}$.
A
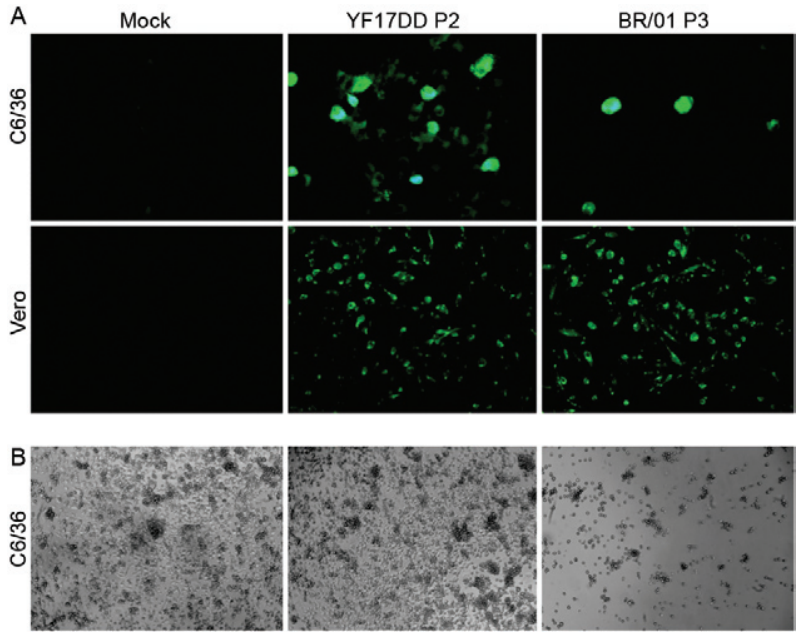

YF BR/01 sample characterization. A: indirect immunofluorescence assay of C6/36 and Vero cells infected with the YF BR/01 sample, a yellow fever positive control (YF17DD P2) or a negative control (mock). Cells were fixed at six (C6/36) or seven (Vero) days post-infection and labelled with anti-yellow fever polyclonal antibody followed by antimouse conjugated to fluorescein isothiocyanate; B: bright field microscopy of C6/36 cells infected or not (mock) with the samples described for A. For all experiments $4 \times 10^{4}$ cells $/ \mathrm{cm}^{2}$ were seeded and were infected $24 \mathrm{~h}$ later with the same amount of virus. In A C6/36 and Vero images were taken with magnification factors of $400 \mathrm{X}$ and $200 \mathrm{X}$, respectively, in B a magnification factor of $100 \mathrm{X}$ was used.

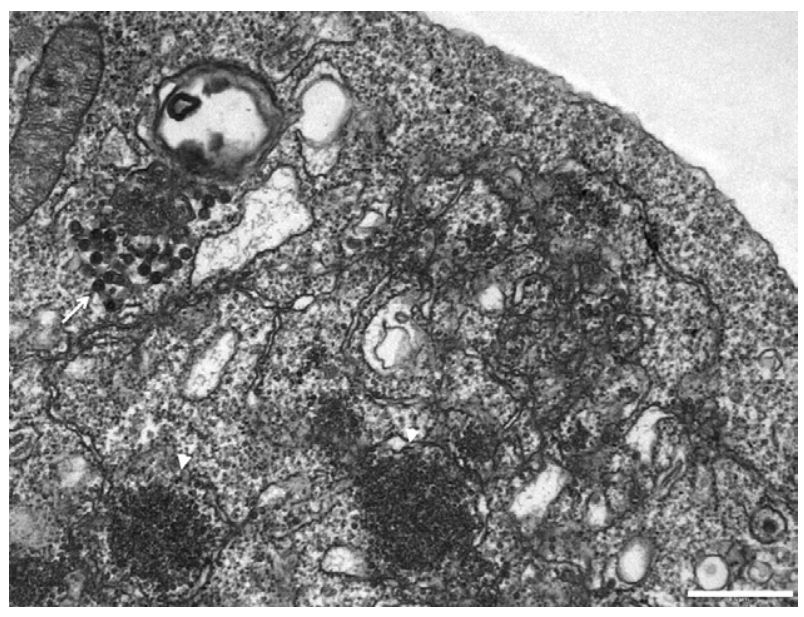

Transmission electron microscopy of C6/36 cells infected with the sample YF BR/01 at $48 \mathrm{~h}$ post-infection. Arrows point to the large particles and arrowheads to an aggregate of smaller particles associated to the endoplasmic reticulum. $\mathrm{Bar}=0.5 \mu \mathrm{m}$.

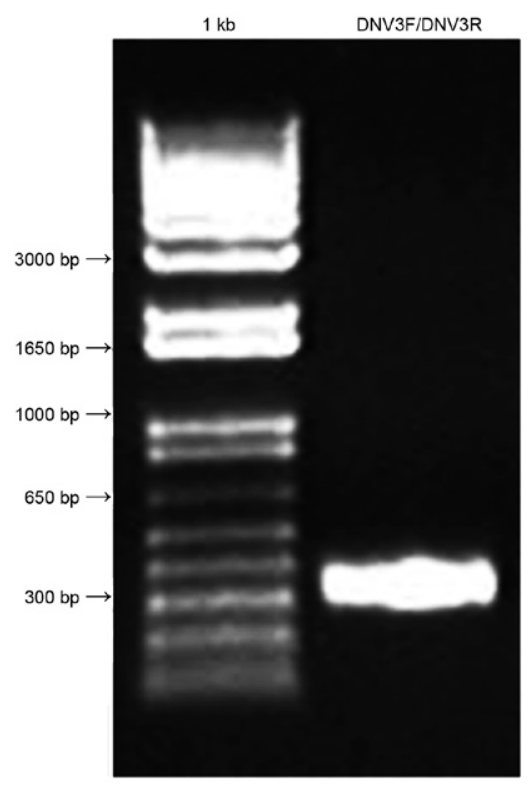

Mosquito densovirus polymerase chain reaction (PCR) product from infected $\mathrm{C} 6 / 36$ cell culture visualized after electrophoresis on a $0.8 \%$ agarose gel. Primers DNV-3F and DNV-3R gave the expected product size of $324 \mathrm{bp}$. The gel was loaded with $250 \mathrm{ng}$ of $1 \mathrm{~Kb}$ Plus DNA Ladder (Invitrogen, Carlsbad, CA, USA) and 10\% (v/v) of the PCR reaction. 
PCR YF

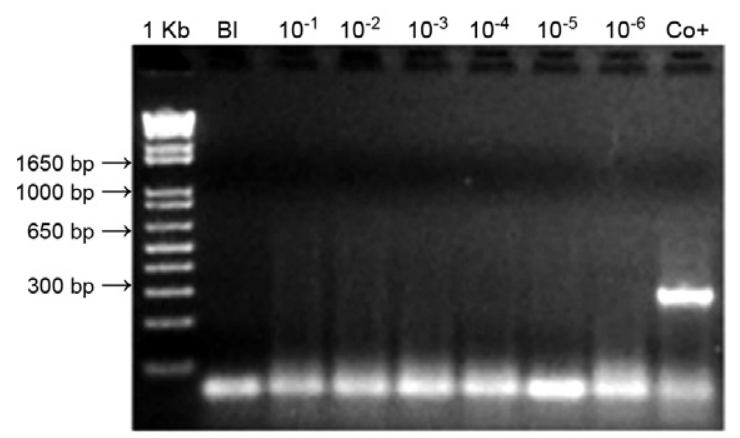

PCR MDV

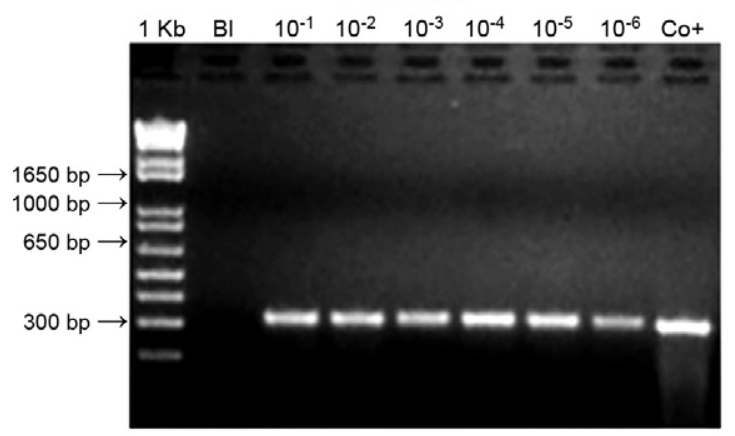

Isolation of mosquito densovirus (MDV). Polymerase chain reaction (PCR) from C6/36 cells infected with different dilutions $\left(10^{-1}-10^{-6}\right)$ of the $\mathrm{YF}$ BR/01 sample that had been pre-incubated with anti-yellow fever polyclonal antibody; products were visualized by electrophoresis on $0.8 \%$ agarose gels. MDV PCR was performed with primers DNV-3F and DNV-3R, giving an expected size of $324 \mathrm{bp}$. Yellow fever (YF) PCR was carried out using primers YF34 and YF21, giving an expected size of $291 \mathrm{bp}$. Gels were loaded with $250 \mathrm{ng}$ of $1 \mathrm{~Kb}$ Plus DNA Ladder and 25\% (v/v) of PCR reactions. Bl: blank; $\mathrm{Co}+$ : positive control.

A

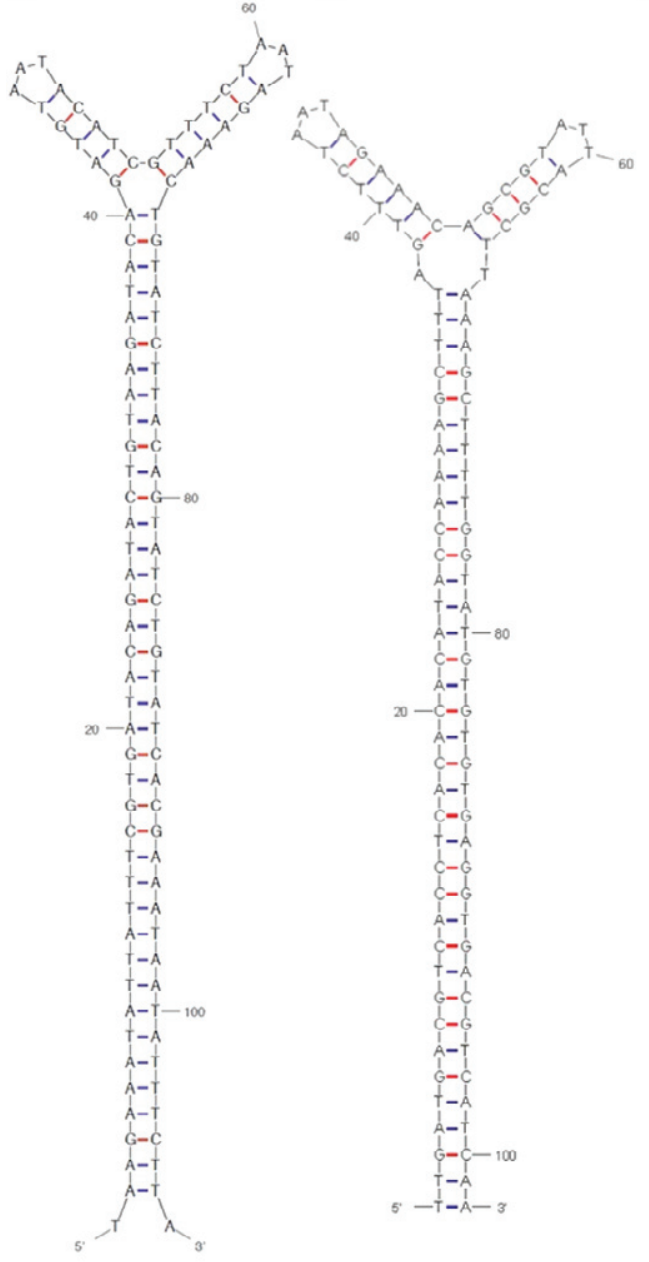

C

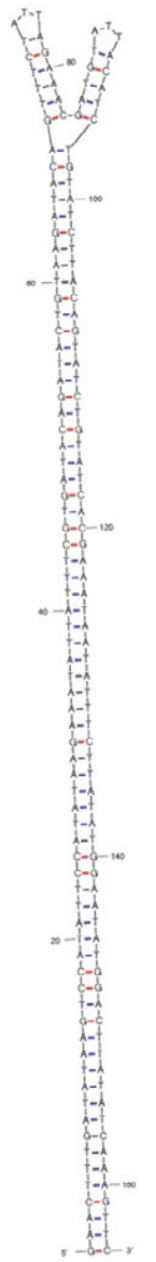

D

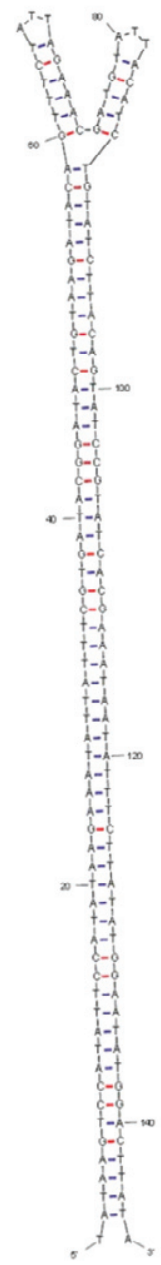

Predicted secondary structures for 5' and 3' ends of the viral genome. 5' ends of (A) BR/07 mosquito densovirus (MDV) and (B) Aedes densonucleosis virus minus strands. 3' ends of (C) BR/07 MDV and (D) Ae. densonucleosis virus minus strands. 


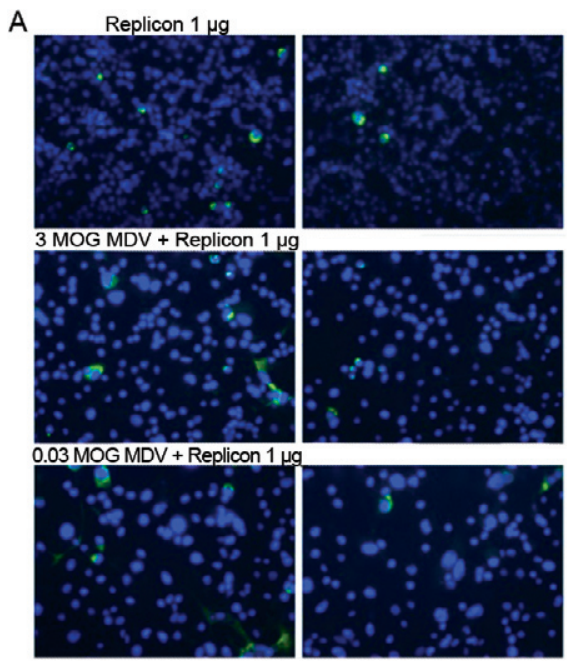

B

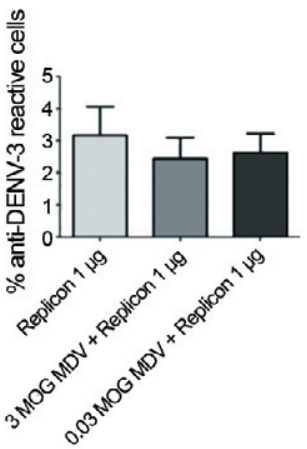

Replicon experiments. A: indirect immunofluorescence assay of C6/36 cultures transfected with $1 \mu \mathrm{g}$ of RNA of the Replicon BR DEN3 290-02 previously infected or not at 0.03 or 3 multiplicity of genomes (MOG) of BR/07 mosquito densovirus (MDV). Cells were fixed and incubated with anti-dengue virus-3 (DENV-3) polyclonal antibody followed by anti-mouse conjugated to fluorescein isothiocyanate. Cell nuclei were stained with 4'-6-diamidine-2-phenyl indole. Images were obtained with a 400X magnification factor. Pictures representative of three experiments; B: estimation of the percentage of anti-DENV-3 reactive cells in C6/36 cultures transfected with $1 \mu \mathrm{g}$ of RNA of the Replicon BR DEN3 290-02 previously infected or not at 0.03 or $3 \mathrm{MOG}$ of $\mathrm{BR} / 07 \mathrm{MDV}$. Results are expressed as means \pm standard deviation of three experiments. 